\title{
ZnO nanoparticles coated by Chitosan-Linoleic acid inhibit the Candida growth and Biofilm formation in vitro
}

ISHAM

Maryam Roudbary ${ }^{\star 1}$, Sanaz barad²,Ayat Nasrollahi Omran³, Mohammad Porgham daryasari ${ }^{3}$, Fatemeh Nikoomanesh ${ }^{4}$

1. Department of Medical Mycology and Parasitology, School of Medicine, Iran University of Medical Sciences, Tehran, Iran

2. Department of Medical Mycology,Faculty of medicine,Tonekabone Branch,Islamic azad university,Tonekabon.Iran

3. Department of Chemistry, Kerman Branch, Islamic Azad University, Kerman, Iran .

4. Department of Medical Mycology, Faculty of Medical Sciences, Tarbiat Modares University, Tehran, Iran.

\section{Objective:}

Candida species is a potential pathogen due to various unique characteristics such as the ability to change morphology, changing from yeast to hyphal, hydrolytic enzymes and forming biofilm which causing many opportunistic fungal disease. Nowadays, various nanoparticles are produced with antifungal properties although several studies have proven the antifungal property of $\mathrm{ZnO}$ nanoparticles. This study has been carried out on reviewing the use of new synthetic component of zinc oxide nanoparticles ( $\mathrm{ZnO}$ NPs) coated by Chitosan-linoleic acid (CS-LA) and to assess Minimum inhibitory concentration (MIC) of nanoparticles on clinical samples and biofilm formation in vitro.

\section{Materials/methods:}

At first the synthesized ZnO NPs coated by CS-LA were identified with X-ray

powder diffraction (XRD), Scanning electron microscope (SEM), Transmission electron microscope (TEM) and Fourier Transform Infrared Spectroscopy analysis (FTIR).Through in vitro tests, the value of MIC and Minimum fungicide concentration (MFC) of nanoparticles and standard and clinical strains of candida were evaluated in comparison with fluconazole as the control group using the CLSI-M27 method. Finally, biofilm formation was studied using MTT assay.

\section{Results:}

The results showed that MIC50 of fluconazole and nanoparticle in clinical strains was $64 \mu \mathrm{g} / \mathrm{ml}$ and $128 \mu \mathrm{g} / \mathrm{ml}$, respectively. The MIC of fluconazole and nanoparticle in C. albicans (ATCC10231) was $8 \mu \mathrm{g} / \mathrm{ml}$ and $32 \mu \mathrm{g} / \mathrm{ml}$ respectively. The MFC of nanoparticle and fluconazole for clinical samples was recorded at similar

level $(128 \mu \mathrm{g} / \mathrm{ml})$. MTT results indicated that the capacity of inhibition of biofilm formation was $43.07 \%$ and $36.68 \%$ by ZnO NPs and fluconazole, respectively. (Table1)1

\section{Conclusions:}

It is concluded that the new synthesized nanoparticle has appropriate efficacy compared with fluconazole in inhibitory activity on C. albicans growth and biofilm formation. As a result, ZnONPs can be introduced as an effective agent for diminishing adhesion capacity of $C$. albicans and biofilm formation. However more in vitro and in vivo investigations are needed.

Key word:ZnO nanoparticle,Candidà,Biofilm inhibition
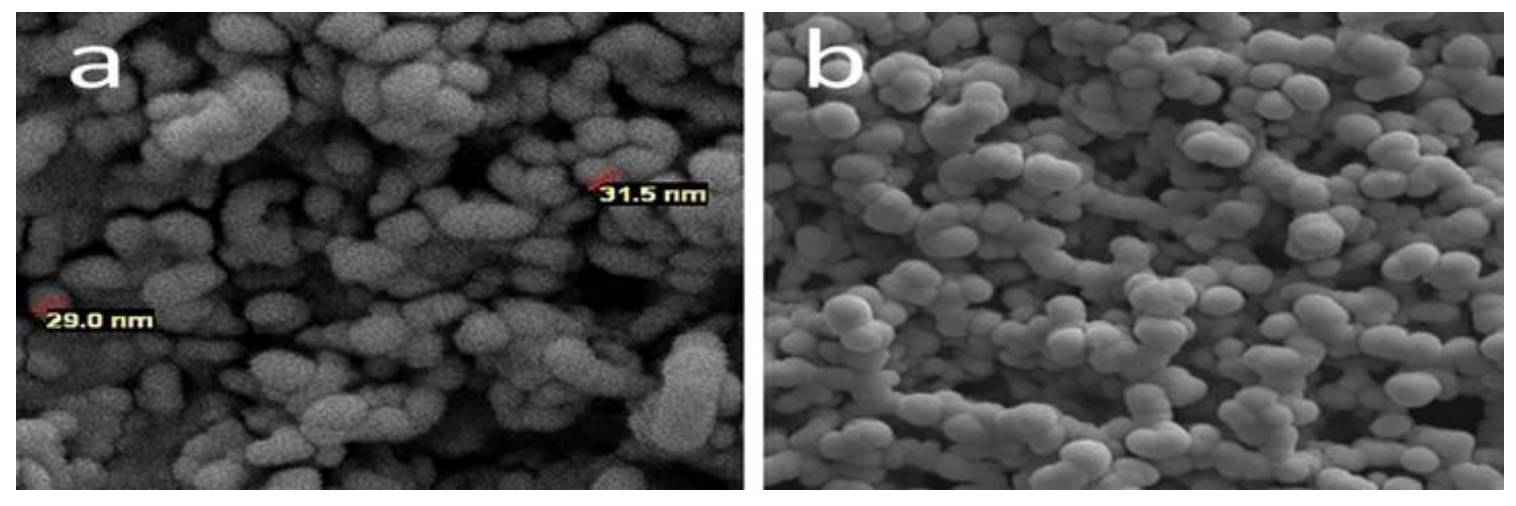

Figure 1. FE-SEM images of $\mathrm{ZnO}$ nanoprticles (a)and (b)

Table1: The value of MIC and MFC of ZnO NPs and fluconazole against C.albicans

\begin{tabular}{|c|c|c|c|c|c|c|c|}
\hline \multirow{2}{*}{ Substance } & \multirow{2}{*}{$\begin{array}{c}\text { MTT } \\
\text { mean of OD }\end{array}$} & \multirow{2}{*}{$\begin{array}{l}\text { Percent inhibition of } \\
\text { biofilm }\end{array}$} & \multicolumn{2}{|c|}{ MIC50* } & \multirow[t]{2}{*}{ MIC Range* } & \multicolumn{2}{|c|}{ MFC } \\
\hline & & & ATCC & Clinical isolates & & ATCC & Clinical isolates \\
\hline Fluconazole & 3.15 & $36.68 \%$ & 8 & 64 & $0.25 \_128$ & 16 & 128 \\
\hline $\mathrm{ZnO} N P s$ & 2.48 & $43.07 \%$ & 32 & 128 & $0.25 \_128$ & 64 & 128 \\
\hline
\end{tabular}

${ }^{*} \mu \mathrm{g} / \mathrm{mL}$ 